\title{
Delineating Origins of Stereocontrol in Asymmetric Pd-Catalyzed $\alpha$-Hydroxylation of 1,3-Ketoesters
}

Alexander M. R. Smith, Henry S. Rzepa, Andrew J. P. White, Denis Billen ${ }^{\xi}$ and King Kuok (Mimi) Hii*

Department of Chemistry, Imperial College London, Exhibition Road, South Kensington, London SW7 2AZ, United Kingdom.

E-mail: mimi.hii@imperial.ac.uk

RECEIVED DATE (to be automatically inserted after your manuscript is accepted if required according to the journal that you are submitting your paper to)

${ }^{\xi}$ Pfizer Animal Health, 333 Portage Street, Kalamazoo, MI 49001, U.S.A. 


\section{Graphical Abstract}

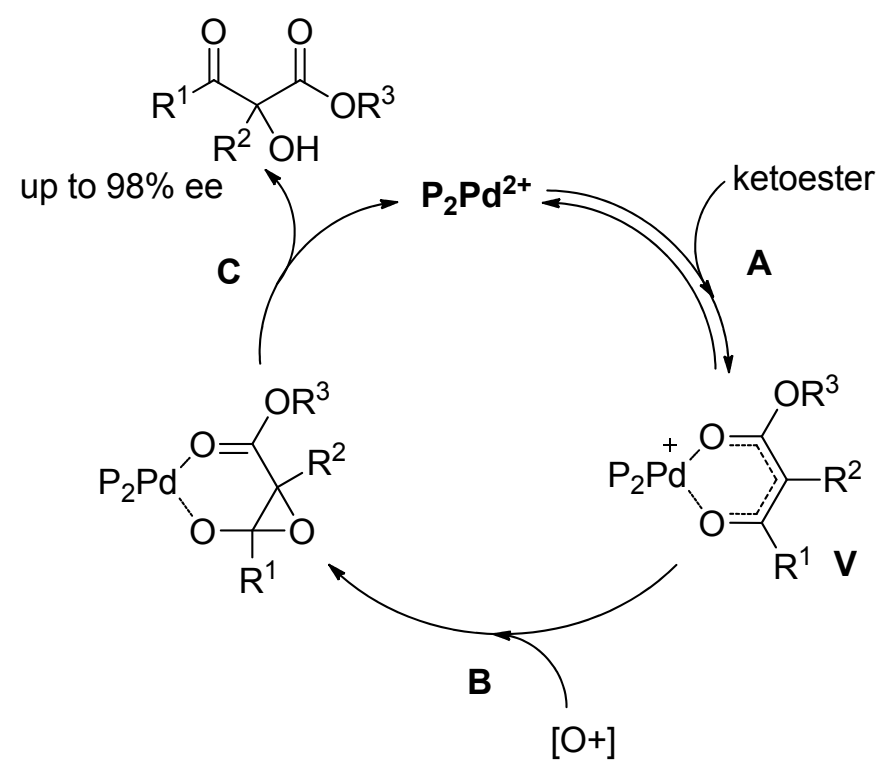

\footnotetext{
Abstract. Systematic studies of reaction conditions and subsequent optimization led to the identification of important parameters for stereoselectivity in the asymmetric $\alpha$-hydroxylation reaction of 1,3-ketoesters. Enantioselectivities of up to $98 \%$ can be achieved for cyclic substrates and $88 \%$ for acyclic ketoesters. Subsequently, the combination of cyclic/acyclic ketoester, catalyst and oxidant was found to have a profound effect on reaction rates. The stereochemistry of the reaction contradicts that observed for other similar electrophilic substitution reactions. This was rationalized by transition state modeling, which revealed a number of cooperative weak interactions between oxidant, ligand and counterion, together with $\mathrm{C}-\mathrm{H} / \pi$ interactions that cumulatively account for the unusual stereoselectivity.
} 


\section{Introduction}

In a Rubottom oxidation reaction, a silyl enol ether undergoes epoxidation by a peracid to furnish an unstable intermediate that hydrolyses to an $\alpha$-hydroxyl ketone (Scheme 1, equation 1). ${ }^{1}$ For 1,3 -dicarbonyl compounds with sufficiently acidic $\alpha$-hydrogens, spontaneous deprotonation occurs upon chelation to Lewis acid catalysts. The resultant enolate complex reacts with electrophilic oxidants, including $m$-CPBA, $\mathrm{H}_{2} \mathrm{O}_{2}$, and $\mathrm{O}_{2}$, to furnish densely functionalized products that are valuable intermediates for organic synthesis (Scheme 1, equation $2)^{2}$

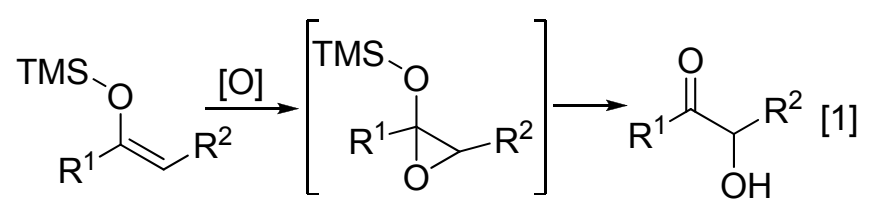

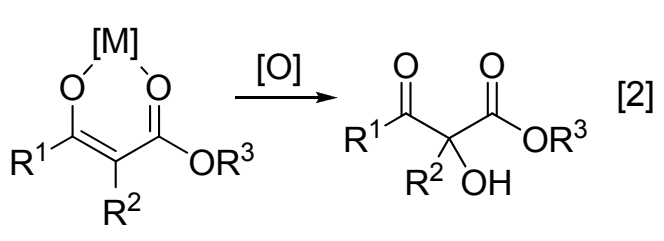

Scheme 1. $\alpha$-Hydroxylation reactions.

A significant body of work by Davis and co-workers has identified a family of oxaziridines (figure 1) as electrophilic oxidants for the hydroxylation of enolates, ${ }^{3,4}$ including the development of a camphorsulfonyl oxaziridine $\mathbf{3}$ as a chiral reagent, which has been employed to great effect in the synthesis of several natural products. ${ }^{5}$<smiles>[R]OS(=O)N1OC1[AlH]</smiles><smiles>CC12ON1c1ccccc1C2=O</smiles>

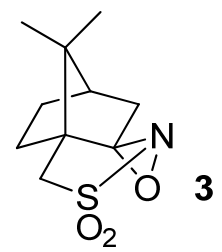

Figure 1. Oxaziridine reagents developed by Davis et al. 
In comparison, the development of catalytic asymmetric hydroxylation reactions has emerged only in recent years. The precedent was set by Togni and Mezzetti in 2004, with the report of TADDOL-Ti complex 4 as a chiral Lewis acid catalyst (figure 2). ${ }^{6}$ Used in combination with racemic $N$-sulfonyl oxaziridine 1 a $\left(\mathrm{Ar}=4-\mathrm{O}_{2} \mathrm{NC}_{6} \mathrm{H}_{4}, \mathrm{R}=\mathrm{Ph}\right)$, $\alpha$-hydroxylation of cyclic and acyclic 1,3-ketoesters can be realized, achieving an impressive $94 \%$ ee for the hydroxylation of tert-butyl cyclopentanone-2-carboxylate. A few years later, Toru and Shibata reported the use of DPFOX complexes of $\mathrm{Zn}$ and $\mathrm{Ni}$ for the hydroxylation of oxindoles and cyclic ketoesters using saccharin-derived oxaziridine 2 as oxidant. $^{7}$

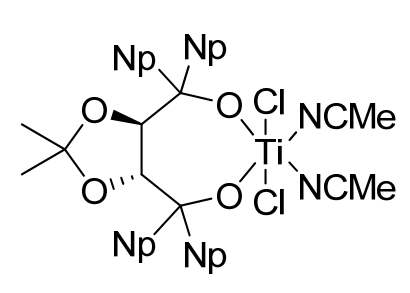

4

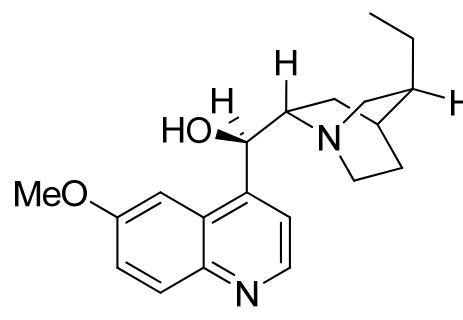

5

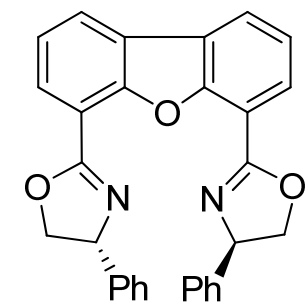

DPFOX

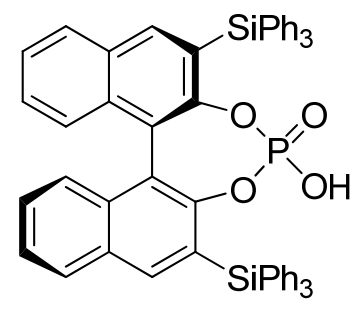

6

Figure 2. Chiral catalysts for asymmetric hydroxylation reactions.

The use of cinchona alkaloid derivatives as chiral organocatalysts for the $\alpha$-hydroxylation of 1,3-ketoesters by organic peroxides was first disclosed in the patent literature by Dupont scientists, ${ }^{8}$ also later by Jørgensen and co-workers, ${ }^{9}$ where moderately high ee's can be attained (up to $80 \%$ using catalyst 5). Recently, a more successful approach was recorded by using a combination of the chiral phosphoric acid 6 and PhNO, ${ }^{10}$ where ee's of up to $98 \%$ can be achieved. With the exception of the Ti catalyst 4 , most of these catalysts were only effective for 
the hydroxylation of cyclic 1,3-ketoesters: acyclic substrates were either unreported, or proceeded with much diminished yields and/or stereoselectivity.

Recently, we reported the use of dimethyldioxirane (DMD) as an effective oxidant for $\alpha$ hydroxylation of 1,3-ketoesters. ${ }^{11}$ In the presence of a dicationic BINAP-palladium catalyst, unprecedented levels of enantioselectivities can be obtained with carbocyclic, heterocyclic and acyclic substrates. Further details of this work are described herein, including the identification of key elements for reactivity and stereocontrol, and the proposal of a novel transition state for $\alpha$-heterofunctionalization of 1,3-ketoesters.

\section{Results and Discussion}

We previously demonstrated the dicationic $\operatorname{Pd}(\mathrm{II})$ complex $(R)$-I to be a highly effective catalyst for the enantioselective addition of aromatic amines to chelating Michael acceptors. ${ }^{12-14}$ Previously, seminal work by Sodeoka and co-workers exploited the chelation of 1,3-ketoesters to catalyst I as a means of stereocontrol in a series of aldol, Mannich, Michael, and related reactions, where excellent levels of enantioselectivity can be achieved. ${ }^{15}$ Concurrently, highly enantioselective $\alpha$-fluorination and $\alpha$-amination of 1,3-ketoesters have also been reported. ${ }^{16-18}$ In light of this, the absence of enantioselective $\alpha$-hydroxylation reactions was striking. Given that this particularly class of $\mathrm{C}-\mathrm{O}$ bond forming reactions is not well-demonstrated in asymmetric catalysis, we decided to explore the application of dicationic $\mathrm{Pd}(\mathrm{II})$ catalysts in these systems.

Screening of oxidants. The cyclic 1,3-ketoester methyl 2-carboxylate indanone (7a) was adopted as a model substrate in the screening of oxidants (Scheme 2, Table 1). Reactions were performed initially with four oxaziridines: 1b, 1c and both isomers of $\mathbf{3}$. While the first two oxidants afforded similar results (entries 1 and 2), neither isomer of the chiral camphor 
oxaziridine was reactive (entry 3), suggesting that the size of the oxidant is important for reactivity. Subsequently, other (smaller) electrophilic oxidants were examined. Peroxides were less reactive and selective than $m$-CPBA (entries 4, 5 and 6), although opposite stereoselectivity was observed when tert-butyl peroxide was used. ${ }^{19}$ When Oxone was employed, the precursor was consumed quickly to give a capricious mixture, from which the expected product was isolated in a low yield (entry 7). Last but not least, the use of dimethyldioxirane (DMD) was also assessed. As the reagent is highly electrophilic, the reaction was conducted at $-20^{\circ} \mathrm{C}$ to suppress the uncatalyzed reaction (vide infra), where comparable level of enantioselectivity to that achieved by $\mathbf{1 b}$ was obtained. Thus, this study identified oxaziridine $\mathbf{1 b}$ and DMD as the best oxidants, as they offered the cleanest and highest conversions.<smiles>CC(=O)C1Cc2ccccc2C1=O</smiles>

$7 a$

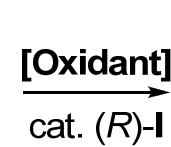

cat. $(R)-\mathbf{I}$

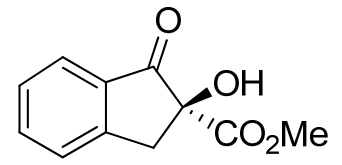

$8 a$

2+

(R)-I<smiles></smiles>

Scheme 2. Initial model system of study.

Table 1 Hydroxylation of $7 \mathbf{a}$ by different oxidants (Scheme 2). ${ }^{a}$

\begin{tabular}{llcccc}
\hline Entry & Oxidant & $\mathrm{t}^{b} / \mathrm{h}$ & $\mathrm{T} /{ }^{\circ} \mathrm{C}$ & Conversion $/ \%^{c}$ & $\mathrm{ee}^{\mathrm{d} / \%}$ \\
\hline 1 & $\mathbf{1 b}$ & 18 & $\mathrm{rt}$ & $100(99)$ & $(S)-(+)$ \\
& $(\mathrm{Ar}=\mathrm{Ph}, \mathrm{R}=p$-tol $)$ & & & & \\
2 & $\mathbf{1 c}$ & 18 & $\mathrm{rt}$ & $100(95)$ & $(S)$ \\
& $\left(\mathrm{Ar}=4-\mathrm{NO}_{2} \mathrm{C}_{6} \mathrm{H}_{4}, \mathrm{R}=p\right.$-tol $)$ & & & &
\end{tabular}




\begin{tabular}{|c|c|c|c|c|c|}
\hline 3 & $\mathbf{3}^{e}$ & 18 & $\mathrm{rt}$ & 0 & \\
\hline 4 & $\mathrm{H}_{2} \mathrm{O}_{2}^{f}$ & 18 & $\mathrm{rt}$ & 0 & \\
\hline 5 & $t-\mathrm{BuOOH}^{g}$ & 18 & $\mathrm{rt}$ & $27(24)$ & $(R)-(-)$ \\
\hline 6 & $m$-СРВА & 18 & rt & $71(64)$ & $(S)$ \\
\hline 7 & Oxone & 18 & $\mathrm{rt}$ & $100(24)^{h}$ & $(S)$ \\
\hline 8 & DMD & 0.5 & -20 & 100 (99) & $(S)$ \\
\hline
\end{tabular}

Ligand Screening. Previously, we have successfully employed $\mathrm{Pd}(\mathrm{OTf})_{2} \cdot 2 \mathrm{H}_{2} \mathrm{O}$ as a catalyst precursor for the generation of dicationic diphosphine-palladium complexes in situ, which facilitated high-throughput ligand screening studies. ${ }^{20}$ Accordingly, this was employed in the current work for the rapid assessment of ligand effects in these hydroxylation reactions. In total, twenty-one phosphine-containing ligands with different structural and electronic features (figure 3) were tested using oxaziridine $\mathbf{1 b}$ as the oxidant, as reactions can be conducted at room temperature. $^{21}$

Although individual rates were not monitored, reactions were essentially complete within $18 \mathrm{~h}$. Among the $C_{2}$-symmetric ligands, chiral diphosphines with axial chirality (BINAP, MeO-ClPHEP and P-Phos) offered virtually identical enantioselectivities (28-30\%). In comparison, a much higher level of selectivity was offered by the planar-chiral $(R)$-Phanephos $(53 \%$ ee of the opposite enantiomer was obtained), while increasing the steric bulk of the phosphine moiety (xylyl-Phanephos) has a detrimental effect (13\% ee). Interestingly, the introduction of a paramethoxy group on the paracyclophane enhanced the ee (MeO-ParaPhos vs xylyl-Phanephos), 
indicating that non- $C_{2}$ symmetric ligands are potentially useful. Planar-chiral diphospholanes based on the ferrocene backbone (FerroTanes) displayed higher levels of selectivity than DuPhos containing stereogenic carbons. The study of $C_{2}$-symmetric ligands concluded with DIOP and SpirOP, which gave practically racemic products.

Next, non- $C_{2}$-symmetric ligands were examined: Diphosphines such as Walphos and Josiphos offered low levels of selectivity (11\% and $8 \%$, respectively), while Stanphos and BoPhoz containing electronically dissimilar P-donors displayed a level of selectivity similar to that of BINAP (25 and 30\% ee, respectively). Enantioselectivities obtained by ligands with different donor groups $(\mathrm{P}-\mathrm{N})$ are particularly interesting. While phosphinooxazoline (PHOX) ligands displayed low levels of enantioselectivity $(<10 \%)$, higher selectivity were achieved with aminophosphine ligands such as Taniaphos (T001, 45\% ee) and MandyPhos (M001, 50\% ee). The selectivity of the latter is dependent on the M:L ratio, which was higher at 2:1 compared to 1:1, suggesting that $\mathrm{P}, \mathrm{N}$-chelation imparts greater stereodifferentiation. Once again, increasing the electronic/steric bulk of the P-substituent led to a decrease in these values (T002 and M002). 


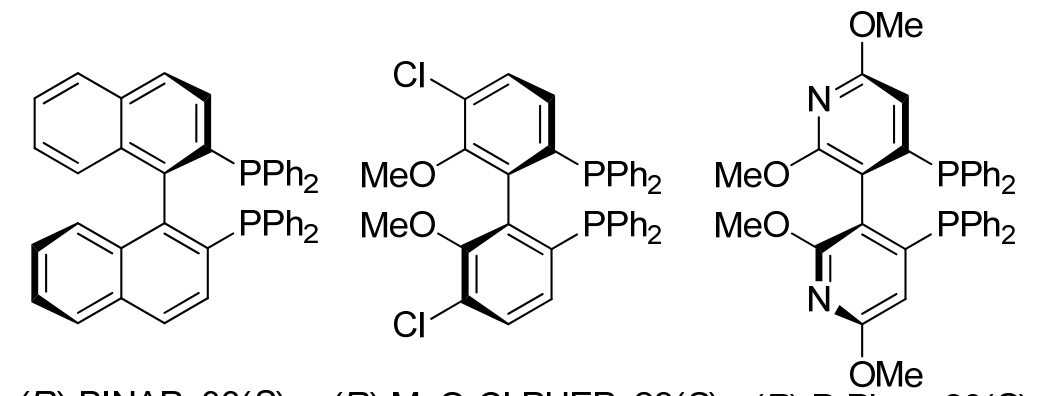

(R)-BINAP, 30(S) (R)-MeO-Cl-PHEP, 28(S) (R)-P-Phos, 29(S)

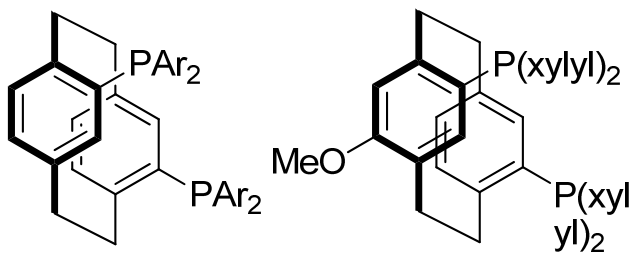

PhanePhos (R)-MeO-ParaPhos, $\mathrm{Ar}=\mathrm{Ph}, 53(R) \quad 38(R)$

$\mathrm{Ar}=\mathrm{xylyl}, 13(R)$<smiles>C[C@H]1CC[C@H](C)P1c1ccccc1P1[C@H](C)CC[C@H]1C</smiles>

$(S, S)-M e-D u P h o s, 5(R)$

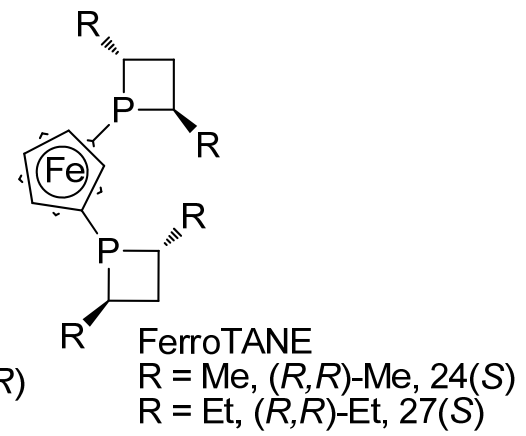<smiles>CC(c1ccccc1-c1ccccc1)P(c1ccccc1)c1ccccc1</smiles>

Walphos, W002, 11(R)<smiles>CC(c1ccccc1P)P(N)(=O)c1ccccc1</smiles><smiles>CC(c1ccccc1Pc1ccccc1)N(C)Pc1ccccc1</smiles>

$(R, R)$-DIOP, $1(R)$

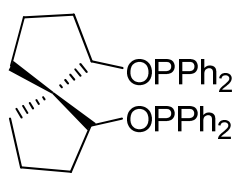

(R)-SpirOP, 4(S)

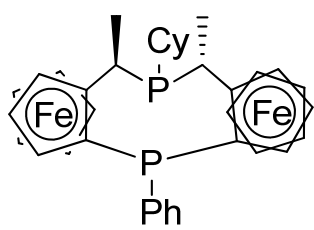

Stanphos, 25(R)

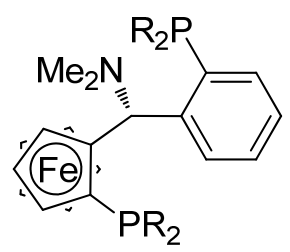

Taniaphos $\mathrm{R}=\mathrm{Ph}, \mathrm{T} 001,45(S)$ $\mathrm{R}=\mathrm{Cy}, \mathrm{T002}, 4(S)$<smiles></smiles>

\section{MandyPhos}

$\mathrm{R}=\mathrm{Ph}, \mathrm{M} 001,22(R), 50(R)^{b}$

$\mathrm{R}=\mathrm{Cy}, \mathrm{M} 002,6(S), 22(R)^{b}$<smiles>[R]C1COC(c2ccccc2P)=N1</smiles>

PHOX

$\mathrm{R}=i-\operatorname{Pr}, 8(S)$

$\mathrm{R}=t-\mathrm{Bu}, 3(\mathrm{~S})$

Figure 3. Ligand screening. Reaction conditions: $10 \mathrm{~mol} \% \mathrm{Pd}(\mathrm{OTf})_{2} \cdot 2 \mathrm{H}_{2} \mathrm{O}, 11 \mathrm{~mol} \%$ ligand, $\mathrm{CH}_{2} \mathrm{Cl}_{2}, 30$ mins, then $\mathbf{7 a}(1 \mathrm{eq})$ and $\mathbf{1 b}(1.2 \mathrm{eq})$ were added successively, r.t. All reactions were complete in $18 \mathrm{~h}\left({ }^{1} \mathrm{H}\right.$ NMR spectroscopy). Ee values determined by chiral HPLC, stereochemistry of major enantiomer provided in parenthesis. ${ }^{b} 5.5 \mathrm{~mol} \%$ ligand. 
The study led us to identify ligand scaffolds that can enable higher levels of selectivity than BINAP, particularly those that contain planar chirality, such as PhanePhos and $\mathrm{P}, \mathrm{N}-$ ligands Taniaphos and Mandyphos. Taking the availability and cost of these ligands into account, only Phanephos was pursued further in the present study. Subsequently, catalyst (R)-II (figure 4) was prepared and its catalytic activity was compared with $(R)$-I in our further work.

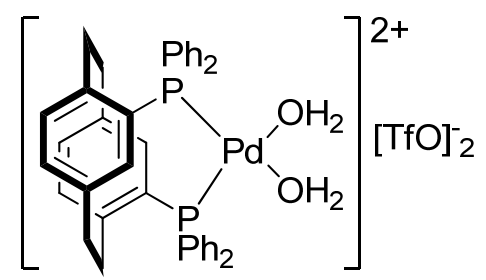

$(R)-$ II

Figure 4. Isolated Phanephos complex $(R)$-II.

Reaction conditions and effect of ester substituent. The performance of catalyst II was examined in the hydroxylation of a series of 1,3-ketoesters derived from indanone (7a-f), containing different ester substituents (Table 2, entries 1-6). By lowering the reaction temperature, there ensued a small but detectable increase in the ee of product 8a, from 56 to $69 \%$ at $-20^{\circ} \mathrm{C}$, at the expense of rate of catalytic turnover (entries 1-4). However, increasing the steric bulk of the ester did not lead to an increase in enantioselectivity as anticipated (entries 5-10). Instead, a decrease to $38 \%$ ee was observed with the tert-butyl ester (entry 12). The same result was obtained using catalyst $\mathbf{I}$, where the hydroxylation of the methyl and tert-butyl esters proceeded with very low levels of enantioselectivity (entries 13-15).<smiles>O=C(O)C1Cc2ccccc2C1=O</smiles>

7

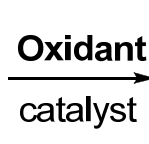

catalyst

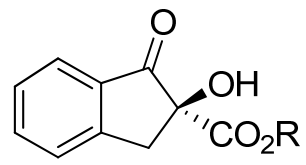

8

$\mathrm{R}=\mathrm{Me}(\mathbf{a}), \mathrm{Et}(\mathbf{b}), i-\operatorname{Pr}(\mathbf{c}), \mathrm{Bn}(\mathbf{d}), t-\mathrm{Bu}(\mathbf{e}), 1-\mathrm{Ad}(\mathbf{f})$

Scheme 3. Hydroxylation of 2-alkyl carboxylate indanones. 
Table $\mathbf{2}$ Effect of catalyst and ester substituent using $\mathbf{1 b}$ as oxidant. ${ }^{a}$

\begin{tabular}{|c|c|c|c|c|c|c|}
\hline Entry & ketoester & catalyst & $\mathrm{T} /{ }^{\circ} \mathrm{C}$ & $\mathrm{t} / \mathrm{h}$ & $\%$ conversion $^{b}$ & $\% \mathrm{ee}^{c}$ \\
\hline 1 & $7 \mathbf{a}$ & II & $\mathrm{rt}$ & 4 & 100 & $56(R)-(-)^{d}$ \\
\hline 2 & & & 0 & 6 & 100 & $52^{e}$ \\
\hline 3 & & & -10 & 7 & 100 & $61^{e}$ \\
\hline 4 & & & -20 & 18 & 100 & $69^{e}$ \\
\hline 5 & $7 \mathbf{b}$ & II & $\mathrm{rt}$ & 4 & $100(62)$ & $50(-)$ \\
\hline 6 & & & -20 & 20 & $100(79)$ & 52 \\
\hline 7 & $7 c$ & II & $\mathrm{rt}$ & 4 & $100(63)$ & $49(-)$ \\
\hline 8 & & & -20 & 20 & $100(73)$ & 54 \\
\hline 9 & $7 d$ & II & $\mathrm{rt}$ & 4 & $100(67)$ & $49(R)-(-)^{d}$ \\
\hline 10 & & & -20 & 20 & $100(69)$ & 63 \\
\hline 11 & $7 e$ & II & $\mathrm{rt}$ & 4 & $100(14)$ & $25(R)-(-)^{d}$ \\
\hline 12 & & & -20 & 20 & $100(66)$ & 38 \\
\hline 13 & $7 \mathbf{a}$ & I & $\mathrm{rt}$ & 4 & $100(99)$ & $30(S)-(+)^{d}$ \\
\hline 14 & $7 e$ & $\mathbf{I}$ & $\mathrm{rt}$ & 2 & 100 & 5 \\
\hline 15 & & & -30 & 24 & 100 & $10(S)-(+)$ \\
\hline \multicolumn{7}{|c|}{$\begin{array}{l}{ }^{a} \text { Reaction conditions: Ketoester } 7 \mathbf{a}-\mathbf{e}(0.123 \mathrm{mmol}) \text {, catalyst }(10 \mathrm{~mol} \%), \mathbf{1 b}(1.2 \text { equiv), } \\
\mathrm{CH}_{2} \mathrm{Cl}_{2} .{ }^{b} \text { Conversion determined by }{ }^{1} \mathrm{H} \mathrm{NMR} \text { spectroscopy, isolated yields (after column } \\
\text { chromatography) given in parenthesis. }{ }^{c} \text { Determined by chiral HPLC. }{ }^{d} \text { Absolute configuration } \\
\text { assigned by comparison with literature data. }{ }^{e} 2.2 \text { equivalents of oxidant. }\end{array}$} \\
\hline
\end{tabular}

Attributing the lack of correlation between the enantioselectivity and the size of the ester substituent to a poor fit of the oxidant with the chiral pocket, the reactions were repeated using DMD as the oxidant, which had previously given comparable results to $\mathbf{1 b}$ in the hydroxylation of 7a (Table 1, entries 2 and 8). The use of DMD can be advantageous, in that both the reagent 
and its side product (acetone) are volatile, which can be evaporated to afford the product in high purity without the need for column chromatography. As it is a powerful oxidant, it is important to suppress competitive uncatalyzed reactions. Thus, the reaction of $\mathbf{7 a}$ and DMD was examined at different temperatures in the absence of the catalyst, establishing the threshold of $-20{ }^{\circ} \mathrm{C}$ for the uncatalyzed process (Table 3, entries 1-3).

Employing catalyst I under these new conditions, a correlation between enantioselectivity and size of the ester substituent can be clearly established, starting from $34 \%$ ee for the methyl ester 7a, raising to $86 \%$ ee for the tert-butyl ester 7e (Table 3, entries 4-9). Exceptions were found with benzyl and adamantyl esters, which afforded lower ee's. Conversions were also extremely rapid; even at $5 \mathrm{~mol} \%$ catalyst loading, reactions were complete within 30 minutes at $-20{ }^{\circ} \mathrm{C}$ (entries 11-16). In contrast, the Phanephos-ligated catalyst II furnished a much lower level of enantioselectivity, and no clear correlation between the enantioselectivity and the ester substituent was observed (entries 17-20).

Table 3 Effect of catalyst and ester substituent using DMD as oxidant. ${ }^{a}$

\begin{tabular}{lllllll}
\hline Entry & ketoester & catalyst & $\mathrm{T} /{ }^{\circ} \mathrm{C}$ & $\mathrm{t} / \mathrm{h}$ & \multicolumn{2}{l}{ \%conversion $^{b} \% \mathrm{ee}^{c}$} \\
\hline 1 & $\mathbf{7 a}$ & none & $\mathrm{rt}$ & 0.17 & 100 & - \\
2 & & -20 & 1 & 6 & - \\
3 & & -78 & 5 & 0 & - \\
4 & $7 \mathbf{a}$ & $\mathbf{I}(10)$ & -20 & 0.5 & $100(99)$ & $39(S)-(+)^{d}$ \\
5 & & & -78 & 5 & 67 & 47 \\
6 & $7 \mathbf{b}$ & $\mathbf{I}(10)$ & -20 & 4 & $100(78)$ & $54(+)$ \\
7 & $7 \mathbf{c}$ & $\mathbf{I}(10)$ & -20 & 4 & $100(79)$ & $60(+)$ \\
8 & $7 \mathbf{I d}$ & $\mathbf{I}(10)$ & -20 & 4 & $100(67)$ & $25(S)-(+)^{d}$
\end{tabular}




\begin{tabular}{|c|c|c|c|c|c|c|}
\hline 9 & $7 e$ & I (10) & -20 & 0.5 & $100(88)$ & $86(S)-(+)^{d}$ \\
\hline 10 & $7 f$ & I (10) & -20 & 0.5 & 100 (99) & $29(S)-(+)^{d}$ \\
\hline 11 & $7 \mathbf{a}$ & I (5) & -20 & 0.5 & $100(95)$ & 26 \\
\hline 12 & $7 b$ & I (5) & -20 & 0.5 & $100(88)$ & 48 \\
\hline 13 & $7 c$ & I (5) & -20 & 0.5 & $100(86)$ & 48 \\
\hline 14 & $7 d$ & $\mathbf{I}(5)$ & -20 & 0.5 & $100(91)$ & 15 \\
\hline 15 & $7 e$ & $\mathbf{I}(5)$ & -20 & 0.5 & $100(95)$ & 85 \\
\hline 16 & $7 f$ & $\mathbf{I}(5)$ & -20 & 0.5 & $100(90)$ & 33 \\
\hline 17 & $7 \mathbf{a}$ & II (10) & -20 & 0.5 & $100(80)$ & 4 \\
\hline 18 & $7 c$ & II (10) & -20 & 0.5 & $100(85)$ & 3 \\
\hline 19 & $7 e$ & II (10) & -20 & 0.5 & $100(90)$ & $26(S)-(+)^{d}$ \\
\hline 20 & $7 f$ & II (10) & -20 & 0.5 & $100(94)$ & $16(R)-(-)^{d}$ \\
\hline
\end{tabular}

This part of the study revealed three key factors for high enantioselectivity: while the ligand effect is important, the choice of oxidant can affect how the particular system responds to changing sizes of the ester substituent.

Reaction scope (cyclic substrates). Subsequently, several cyclic carbo- and hetero-cyclic substrates 9-12 (figure 5) were subjected to the optimized reaction conditions (Table 4). Compared to the indanone-derived substrates 7a-e, hydroxylation of the tetralone-derived substrate 9a-c proceeded with very low enantioselectivity $(<20 \%)$, even at $10 \mathrm{~mol} \%$ catalytic loading (entries 1-3). On the other hand, removal of the aromatic ring from the substrate led to a better result, as $66 \%$ ee was obtained with the cyclohexanone derivative 11 (entry 4). This applied equally to the cyclopentanone derivatives, as the hydroxylation of $\mathbf{1 0 a}-\mathbf{c}$ proceeded with 
much higher enantioselectivities than their corresponding indanone esters $\mathbf{7 b}, \mathbf{7 d}$ and $\mathbf{7 e}$ (Table 4, entries 5-7 vs Table 3, entries 12, 14 and 15). Particularly pleasing is the result obtained for compound 17c (98\% ee), matching the best ee recorded for this substrate. ${ }^{6,710}$ Accordingly, hydroxylation of $N$-heterocycles 12, 13 and $\mathbf{1 4}$ also offered good to excellent enantioselectivities, thus providing a route to hydroxylated pyrrolidine rings (entries 8-13). The nature of the protecting group used in heterocycle 12 proved to be important - the Moc-protected product (18a) was unstable to isolation, resulting in a low yield of the product. In comparison, much better yields were obtained with Cbz- and Boc-protected $\mathbf{1 8 b}$ and 18c. With the $N$-heterocycles 13a and $13 \mathbf{b}$, the reduced acidity of the $\alpha$-proton led to a slower reaction. This was overcome by raising the temperature to $0{ }^{\circ} \mathrm{C}$, to afford the hydroxylated product in excellent yields. The best selectivity was attained for the Boc-protected 19b ( $96 \%$ ee).

Last but not least, a series of succinimide derivatives 14a-d were also examined. Good ee's of up to $87 \%$ can be achieved - the planar nature of the cyclic imides may be the cause of the slightly lower enantioselectivity. The products were found to be highly crystalline as homochiral conglomerates, e.g. the $N$-PMB derivative $20 \mathrm{~b}$ can be rendered optically pure after a single recrystallization from ethyl acetate-hexane.

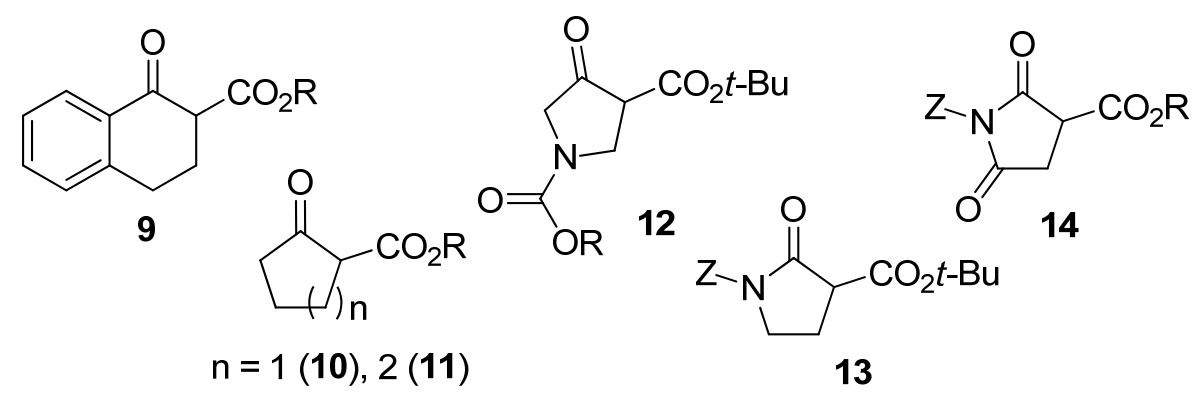

Figure 5. Cyclic carbo- and hetero-cyclic substrates examined in this work. 
Table 4 Asymmetric hydroxylation of carbo- and hetero-cyclic 1,3-ketoesters. ${ }^{a}$

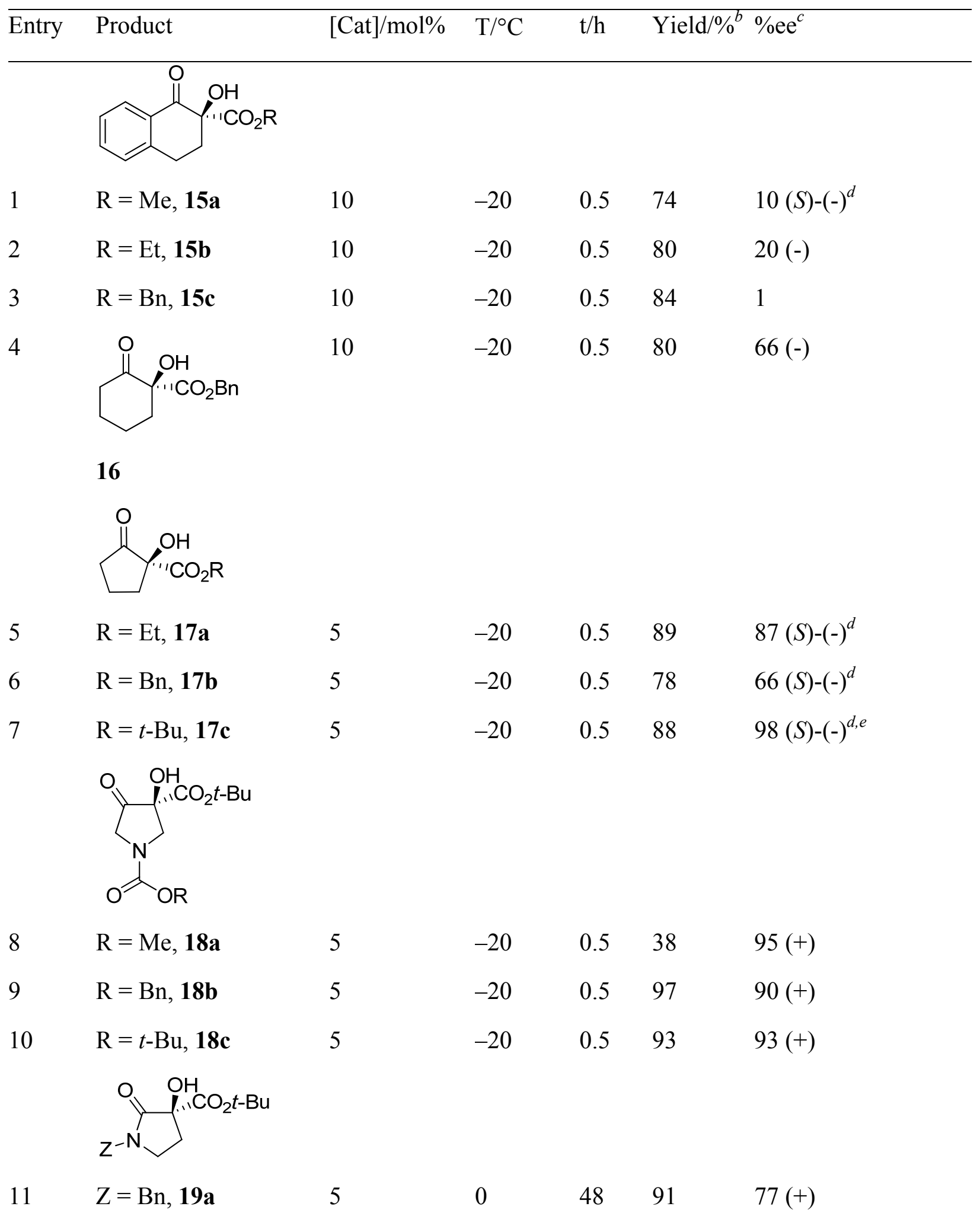




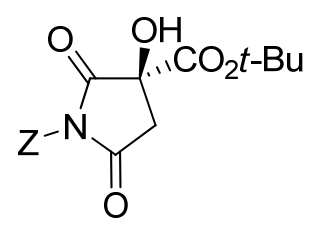

$$
13 \quad \mathrm{Z}=\mathrm{Bn}, \mathbf{2 0 a}
$$

$14 \quad Z=$ PMB, 20b

$15 \mathrm{Z}=t-\mathrm{Bu}, 20 \mathrm{c}$

$16 \quad Z=$ Boc, 20d
5

10

5

5

0

$$
-10
$$

0

0

$\begin{array}{lll}20 & 99 & 83(+) \\ 1 & 92 & 87(S)-(+)^{f} \\ 16 & 94 & 68(+) \\ 4 & 92 & 49^{g}\end{array}$

${ }^{a}$ Reaction conditions: Ketoester $(0.123 \mathrm{mmol})$, catalyst $(R)-\mathbf{I}(5 \mathrm{~mol} \%)$, DMD $(0.7$ $\mathrm{M}$ in acetone, 1.2 equiv), $\mathrm{CH}_{2} \mathrm{Cl}_{2}$. ${ }^{b}$ Isolated yield. All reactions were complete within the given time $\left({ }^{1} \mathrm{H}\right.$ NMR spectroscopy). ${ }^{c}$ Determined by chiral HPLC. ${ }^{d}$ Absolute configuration assigned by comparison to literature data. ${ }^{e}$ Determined by chiral GC. ${ }^{f}$ Determined by X-ray crystallography (vide infra). ${ }^{g}$ Optical rotation was too small to be measured.

\section{Hydroxylation of acyclic ketoesters: Unusual match/mismatch effect of catalyst and}

oxidant. Asymmetric $\alpha$-hydroxylation of acyclic 1,3-ketoesters are rare, presumably due to the operation of competitive processes, e.g. Bayer-Villiger rearrangement, decarboxylation. ${ }^{4,22}$ Prior to our report, only the Ti complex 4 can achieve notable yields and ee values (up to $84 \%$ ). ${ }^{6}$ Recently, an alternate approach to these compounds has also been achieved by the asymmetric alkylation of O-protected $\alpha$-hydroxyl- $\beta$-keto esters. $^{23,24}$

$$
\begin{array}{ll}
\mathrm{R}^{1} & \mathrm{R}^{1}=\mathrm{Me}, \mathrm{R}^{2}=\mathrm{Bn}(\mathbf{2 1}) \\
\mathrm{R}^{1} & \mathrm{R}^{1}=\mathrm{R}^{2}=\mathrm{Me}, \mathrm{R}_{3}=\mathrm{Bn}(\mathbf{2 2}) \\
\mathrm{R}^{1}=\mathrm{Ph}, \mathrm{R}^{2}=\mathrm{Me}, \mathrm{R}^{3}=\mathrm{Et}(\mathbf{2 3}) \\
\mathrm{R}^{1}=\mathrm{Me}, \mathrm{R}^{2}=\mathrm{Ph}, \mathrm{R}_{3}=\mathrm{Et}(\mathbf{2 4})
\end{array}
$$

Figure 6. Acyclic 1, 3-ketoester substrates

Hydroxylation of a series of alkyl 2-benzylacetoacetate 21a-d and commercially available 22 and 24 (figure 6) proceeded slowly under Pd catalysis (Table 5, entries 1-3). Significantly, 
competitive side reactions were not observed, which allowed for increases in the amount of oxidant, catalyst loading $(20 \mathrm{~mol} \%)$ and reaction temperature $\left(0^{\circ} \mathrm{C}\right)$ to achieve acceptable yields (entries 1-3 vs 4-7). Encouragingly, up to $88 \%$ with the tert-butyl ester 21c (entry 6), which is the highest recorded for the $\alpha$-hydroxylation of substrates. On the other hand, $\alpha$-aryl substituted ketoesters are insufficiently active (entry 9).

Table 5 Hydroxylation of acyclic ketoesters catalysed by $\mathbf{I}$, using DMD as oxidant. ${ }^{a}$

\begin{tabular}{|c|c|c|c|c|c|}
\hline Entry & Product & {$[\mathrm{I}] / \mathrm{mol} \%$} & $\mathrm{t} / \mathrm{h}$ & Yield/\% ${ }^{b}$ & ee $/ \%{ }^{c}$ \\
\hline 1 & $\mathrm{R}=\mathrm{Me}, \mathbf{2 5 a}$ & 10 & 18 & 15 & $54(+)$ \\
\hline 2 & $\mathrm{R}=\mathrm{Et}, \mathbf{2 5} \mathbf{b}$ & 10 & 20 & 49 & $61(S)-(+)^{d}$ \\
\hline 3 & $\mathrm{R}=t-\mathrm{Bu}, \mathbf{2 5 c}$ & 10 & 48 & 8 & $86(S)-(+)^{d}$ \\
\hline 4 & $\mathrm{R}=\mathrm{Me}, \mathbf{2 5 a}$ & 20 & 48 & 62 & $65^{e}$ \\
\hline 5 & $\mathrm{R}=\mathrm{Et}, \mathbf{2 5} \mathbf{b}$ & 20 & 48 & 65 & $62^{e}$ \\
\hline 6 & $\mathrm{R}=t-\mathrm{Bu}, \mathbf{2 5 c}$ & 20 & 48 & 55 & $88^{e}$ \\
\hline 7 & $\mathrm{R}=\mathrm{Bn}, \mathbf{2 5 d}$ & 20 & 48 & 52 & $32(+)^{e}$ \\
\hline 8 & 26 & 10 & 20 & 54 & $37(-)$ \\
\hline 9 & 27 & 10 & 20 & 0 & - \\
\hline
\end{tabular}

${ }^{a}$ Reaction conditions: Ketoester $(0.123 \mathrm{mmol})$, catalyst I, DMD (0.7 M in acetone, 1.2 equiv.), $\mathrm{CH}_{2} \mathrm{Cl}_{2}, \quad 0 \quad{ }^{\circ} \mathrm{C} .{ }^{b}$ Isolated yield after column 
chromatography. ${ }^{c}$ Determined by chiral HPLC. ${ }^{d}$ Absolute configuration assigned by comparison with reported data. ${ }^{e} 2$ equivalents of DMD.

Conversely, oxaziridine 1b was ineffective for the hydroxylation of acyclic ketoesters - very little product formation can be obtained using $10 \mathrm{~mol} \%$ of catalyst I or II. However, catalytic activity was restored by using dimeric $\mu$-hydroxy-palladium complexes III and IV (Scheme 4), prepared by the reaction of catalyst I with $0.07 \mathrm{M}$ sodium hydroxide, ${ }^{25}$ or generated in situ by the addition of molecular sieves to $\mathbf{I I},{ }^{26}$ respectively. Using the basic form of these catalysts, good conversions of acyclic ketoesters can be attained using $\mathbf{1 b}$ as the oxidant (Table 5). In all cases, reactions were complete overnight, to afford products of low to moderate level of selectivity.

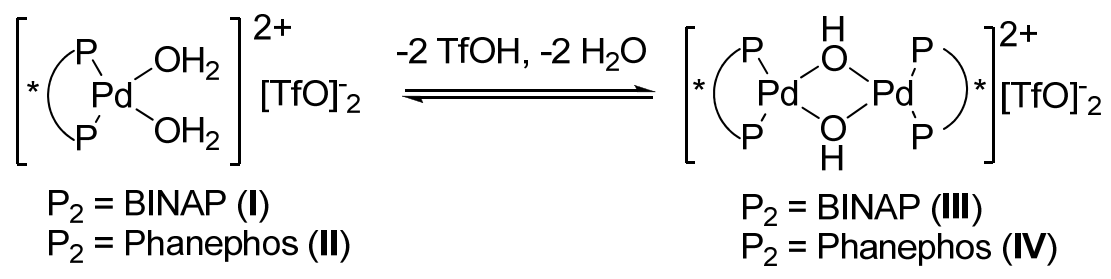

Scheme 4 Formation of dimeric $\mu$-hydroxy-palladium complexes III and IV.

Table 6 Hydroxylation of acyclic ketoesters by $\mathbf{1 b}$ catalyzed by dimeric complexes III and IV.

\begin{tabular}{llllll}
\hline Entry & Product & catalyst & t/h & Yield $/ \%^{b}$ & ee $/ \%^{c}$ \\
\hline & & & & & \\
1 & $\mathrm{R}=\mathrm{Me}(\mathbf{2 5 a})$ & III & 18 & 74 & $48(+)$ \\
2 & IV & 18 & 48 & $9(-)$ \\
3 & $\mathrm{R}=\operatorname{Et}(\mathbf{2 5 b})$ & III & 6 & 58 & $43(S)-(+)$
\end{tabular}




\begin{tabular}{|c|c|c|c|c|c|}
\hline 4 & & IV & 18 & 45 & $14(-)$ \\
\hline 5 & $\mathrm{R}=t-\mathrm{Bu}(\mathbf{2 5 c})$ & III & 18 & 45 & $8(S)-(+)$ \\
\hline 6 & $\mathrm{R}=\mathrm{Bn}(\mathbf{2 5 d})$ & III & 18 & 68 & $16(+)$ \\
\hline 7 & 26 & III & 4 & 71 & 6 \\
\hline 8 & & IV & 18 & 98 & 5 \\
\hline 9 & $\mathrm{R}=\mathrm{Et}(\mathbf{2 7})$ & III & 52 & 29 & 1 \\
\hline 10 & & IV & 18 & 19 & $27(-)$ \\
\hline 11 & $\mathrm{R}=\operatorname{Et} \mathbf{( 2 8 a )}$ & III & 48 & 38 & $29(-)$ \\
\hline 12 & & IV & 48 & 56 & $14(+)$ \\
\hline 13 & $\mathrm{R}=t-\mathrm{Bu}(\mathbf{2 8 b})$ & III & 68 & 60 & $16(-)$ \\
\hline
\end{tabular}

${ }^{a}$ Reaction conditions: Ketoester ( $\left.0.123 \mathrm{mmol}\right)$, catalyst (10 mol\%), $\mathbf{1 b}$ (1.2 equiv), $\mathrm{CH}_{2} \mathrm{Cl}_{2}$, r.t. ${ }^{b}$ Isolated yield after column chromatography. Starting material was consumed (TLC or ${ }^{1} \mathrm{H}$ NMR). ${ }^{c}$ Determined by chiral HPLC. Absolute configuration assigned by comparison of optical rotation with reported value.

Perplexingly, DMD did not afford any product from these substrates using either catalysts III or IV. So it appears that catalytic activity towards achiral ketoesters is only afforded by particular combinations of catalyst and oxidant. This was examined further by monitoring reactions of 21a, using catalyst I or II with oxaziridine 1b or DMD as oxidant under comparable conditions, i.e. maintaining the same reactant concentrations and catalyst loading (figure 7). At 
ambient temperature, the evaporation of volatile DMD led to incomplete conversion. Nevertheless, it is clear that the reaction performed with DMD/catalyst I is slightly faster than the reaction using oxaziridine $\mathbf{1 b}$ /catalyst III (based on initial rates), while other combinations of catalyst and oxidant were very slow.

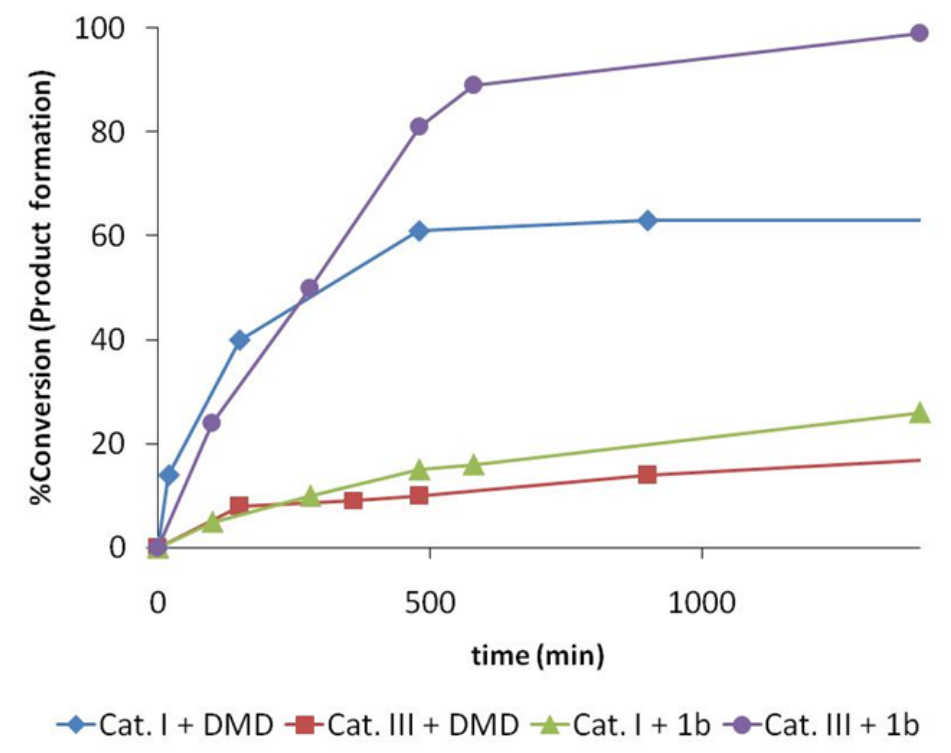

Figure 7. Rates of hydroxylation of 21b with different combinations of catalyst and oxidant (determined by ${ }^{1} \mathrm{H}$ NMR spectroscopy, see Supporting Information). Reaction conditions: 21b $\left(0.123 \mathrm{mmol}\right.$ ), oxidant (1.2 eq), $10 \mathrm{~mol} \%$ I or $5 \mathrm{~mol} \%$ III, $\mathrm{CH}_{2} \mathrm{Cl}_{2}$ (total reaction volume $=3.09$ $\mathrm{mL})$, r.t.

Formation of the Pd-enolate complex. Coordination of 1,3-dicarbonyl compounds to dicationic (diphosphine)palladium(II) complexes is a well-established process. A number of such palladium-enolate complexes have been characterized spectroscopically and also by X-ray crystallography. ${ }^{27,28}$ Chelation of 1,3-dicarbonyl compounds to complex $\mathbf{I}$ is accompanied by the formation of an equivalent of triflic acid (Scheme 5, equation 1), while the chelation to III is promoted by the deprotonation of the $\alpha$-hydrogen by the basic $\mathrm{Pd}-\mathrm{OH}$ moiety (Scheme 5, equation 2). This unique Brønsted acidity or basicity associated with catalysts I and III is often crucial for catalytic activity. ${ }^{29,30}$ 


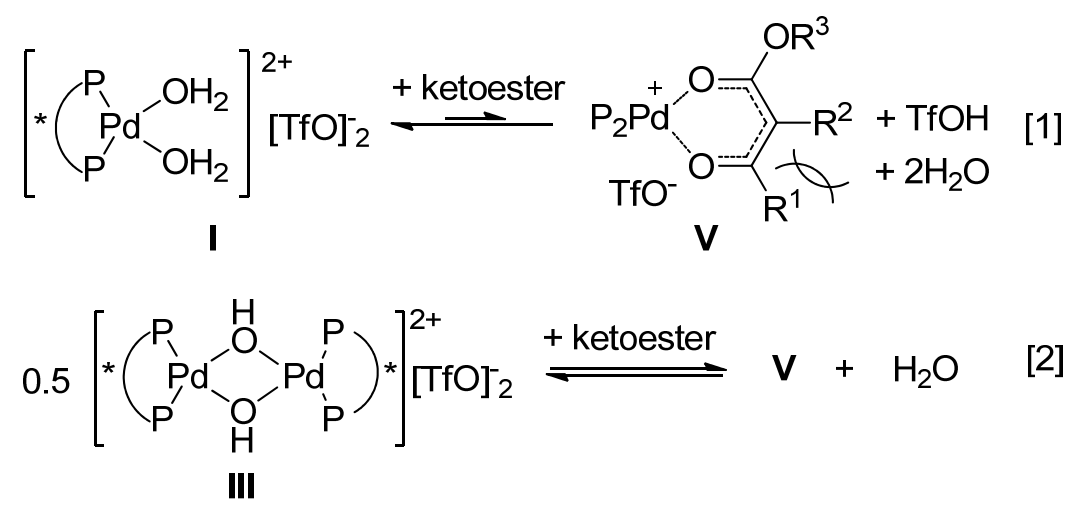

Scheme 5 Formation of palladium-enolate complex $\mathbf{V}$ from I and III.

In our preliminary communication, ${ }^{11}$ we showed that the addition of 10 equivalents of cyclic ketoester 7a to complex III led to the formation of the palladium-enolate complex $\mathbf{V}$, indicated by an AB pattern in the ${ }^{31} \mathrm{P}$ NMR spectrum $\left(\delta_{\mathrm{P}}+29.7,34.7, J=27.5 \mathrm{~Hz}\right) .{ }^{31}$ Reactions of acyclic ketoesters are generally slower. This is attributed to difficulty in the formation of the activated complex $\mathbf{V}$ due to steric interactions between adjacent $\mathrm{R}^{1}$ and $\mathrm{R}^{2}$ substituents, resulting from the planar geometry necessary for chelation (Scheme 5). Thus, the binding of the acyclic substrate 21b to catalysts I and III was also examined by ${ }^{31} \mathrm{P}$ NMR spectroscopy for comparison (figure 8). In contrast to the earlier experiment, chelate formation between III and $\mathbf{2 1 b}$ was observable only in the presence of 200 equivalents of the acyclic ketoester $\left(\delta \mathrm{P}+29.2,+33.4, J=37 \mathrm{~Hz} ; \mathrm{M}^{+}\right.$ $=947$, i.e. the binding of acyclic ketoesters to catalyst III is at least 20 times weaker than the binding of cyclic substrates. As expected, the binding of $\mathbf{2 1 b}$ to complex $\mathbf{I}$ is further hampered by an unfavorable equilibrium (formation of a strong acid), and was barely detectable under the same conditions. 


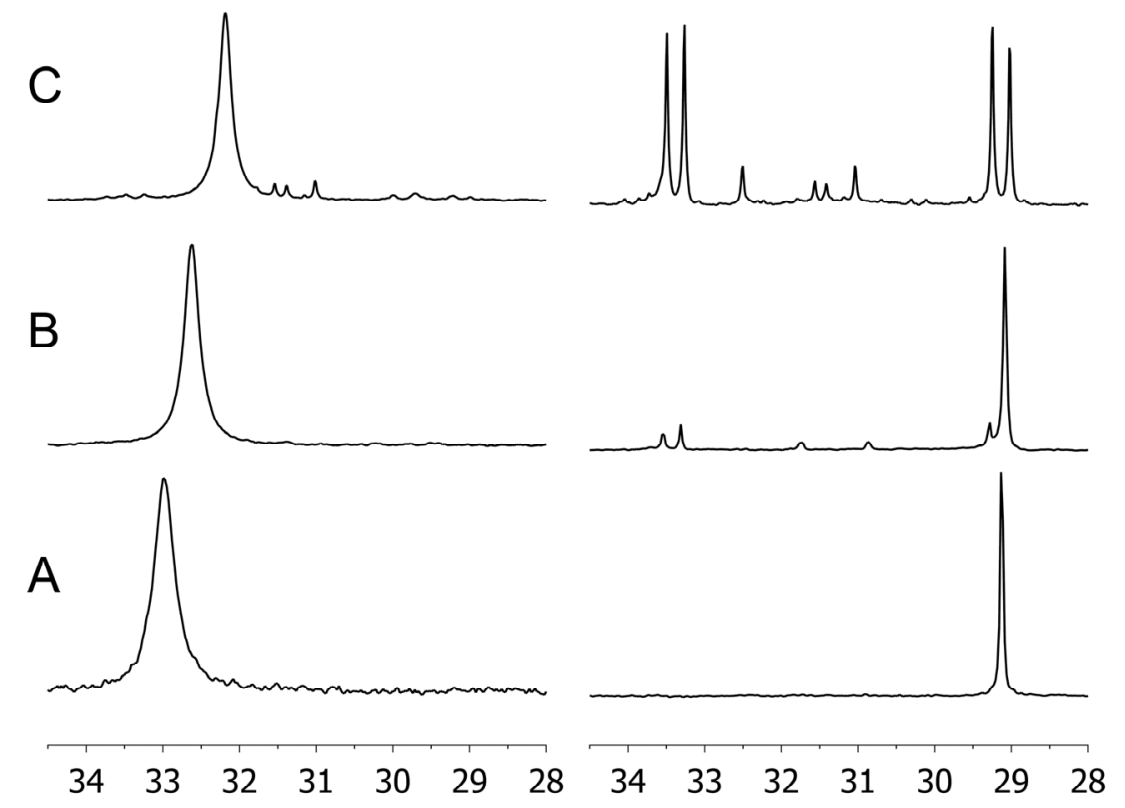

Figure 8. ${ }^{31} \mathrm{P}$ NMR spectra generated from mixtures of complex I (left) and III (right) in $\mathrm{CDCl}_{3}$, with 0 (A), 10 (B) and 200 (C) equivalent(s) of $\mathbf{2 1 b .}$

These binding studies highlighted important aspects of these reactions: If the formation of the Pd-enolate complex is the only mode of activation (and turnover-limiting), reactions catalyzed by complex III should be faster than those catalyzed by complex I. However, this applies only when the oxaziridine $\mathbf{1 b}$ was employed as the oxidant, while the opposite is true when DMD was used (figure 7). The same trends were also observed for the hydroxylation of cyclic ketoesters; while there is no detectable difference between reactions catalyzed by I or III when $\mathbf{1 b}$ was used as the oxidant (Table 7, entries 1 and 2), the reaction with DMD was slower when catalyst III was employed (entries 3 and 4). This suggests that triflic acid plays an important role in reactions involving DMD, but not $\mathbf{1 b}$. It is worth noting that the same ee value was obtained using a specific oxidant, irrespective of the catalyst used. 
Table 7 Comparison of catalyst and oxidants in the hydroxylation of $\mathbf{7 a}{ }^{a}$

\begin{tabular}{llllll}
\hline Entry & Catalyst & oxidant & $\mathrm{T} /{ }^{\circ} \mathrm{C}$ & $\mathrm{t} / \mathrm{h}$ & Conversion (ee)/\% \\
\hline 1 & I & $\mathbf{1 b}$ & $\mathrm{rt}$ & 2 & $100(30)$ \\
2 & III & $\mathbf{1 b}$ & $\mathrm{rt}$ & 2 & $100(30)$ \\
3 & I & DMD & -20 & 0.5 & $100(34)$ \\
4 & III & DMD & -20 & 2 & $41(35)$ \\
\multicolumn{5}{l}{ Catalytic conditions are as described previously in Tables 2 and 4. } \\
\multicolumn{5}{l}{}
\end{tabular}

Catalytic cycles and possible turnover-limiting steps. Based on these observations, working models for these reactions were constructed. For reactions employing DMD (Scheme 6), we established that reactions are faster using catalyst I (entries 3 and 4 in Table 7, and figure 7), even though the formation of the activated palladium-enolate complex is less favored (figure 8); hence, step A is unlikely to be turnover-limiting in this system. Following electrophilic addition (step B), the epoxide intermediate VII presumably rearranges to the putative alkoxide complex VIII (step C). Product release/catalytic turnover will require protonolysis of this intermediate (step D), which is expected to be more facile in the presence of triflic acid. We propose this as the rate-limiting step for reactions involving DMD. Conversely, when oxaziridine $\mathbf{1 b}$ was used as the oxidant, reactions using catalyst III is faster than that mediated by catalyst I. As this corresponds to the relative binding affinities of the ketoester to the Pd complexes, step A is likely to be turnover-limiting in this system. 


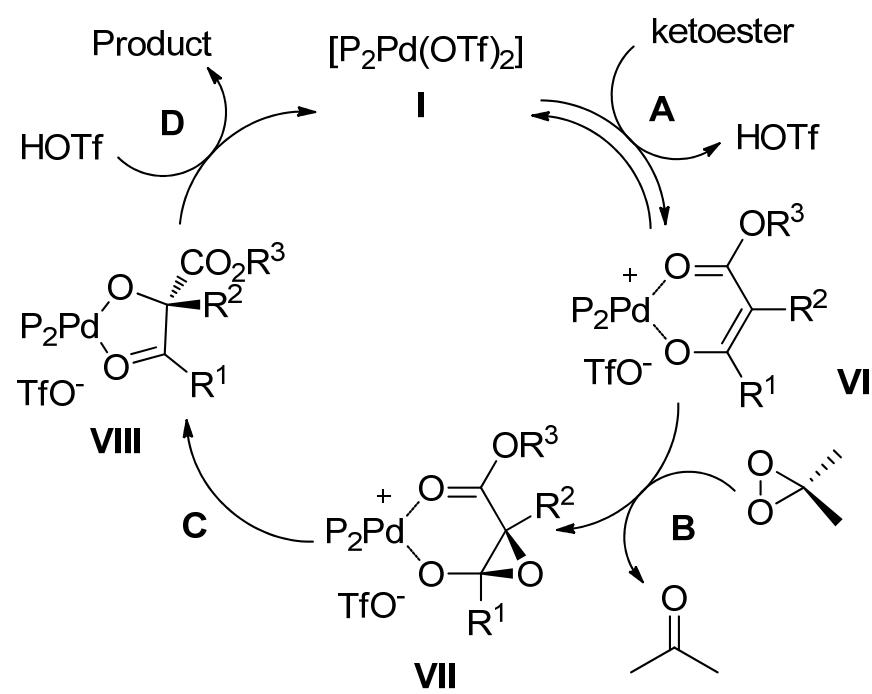

Scheme 6. Postulated catalytic cycle using DMD and catalyst I.

This switch of rate-limiting step between the two systems has significant implications for future development of this work. We speculate that this could be due to: i) an interaction between DMD and triflic acid, such as H-bonding, that enhances the rate of step A; and/or ii) product release is more spontaneous using $\mathbf{1 b}$ as the oxidant, emolliating the effect of triflic acid on step D, which will imply some interaction between the oxidant (or its imine byproduct) with VII or VIII, making them unstable.

Origins of Stereochemistry. Catalyst $(R)-\mathbf{I}$ and related complexes have been used previously in enantioselective $\alpha$-fluorination and $\alpha$-amination of 1,3 -ketoesters. ${ }^{16,18,32}$ In all cases, electrophilic attack occurred preferentially from the Re face of the coordinated enolate (Scheme 7). This was rationalized by the binding of the enolate to the dicationic palladium center, which causes the ester group to occupy the least hindered quadrant of the coordination sphere, thereby hindering the approach of the electrophile from the $S i$ face. ${ }^{16}$ The stereochemical model was also 
applied to related $\mathrm{C}-\mathrm{C}$ bond forming reactions. ${ }^{30} \mathrm{In}$ all cases, the major enantiomer results from an electrophilic addition on the $R e$ face.

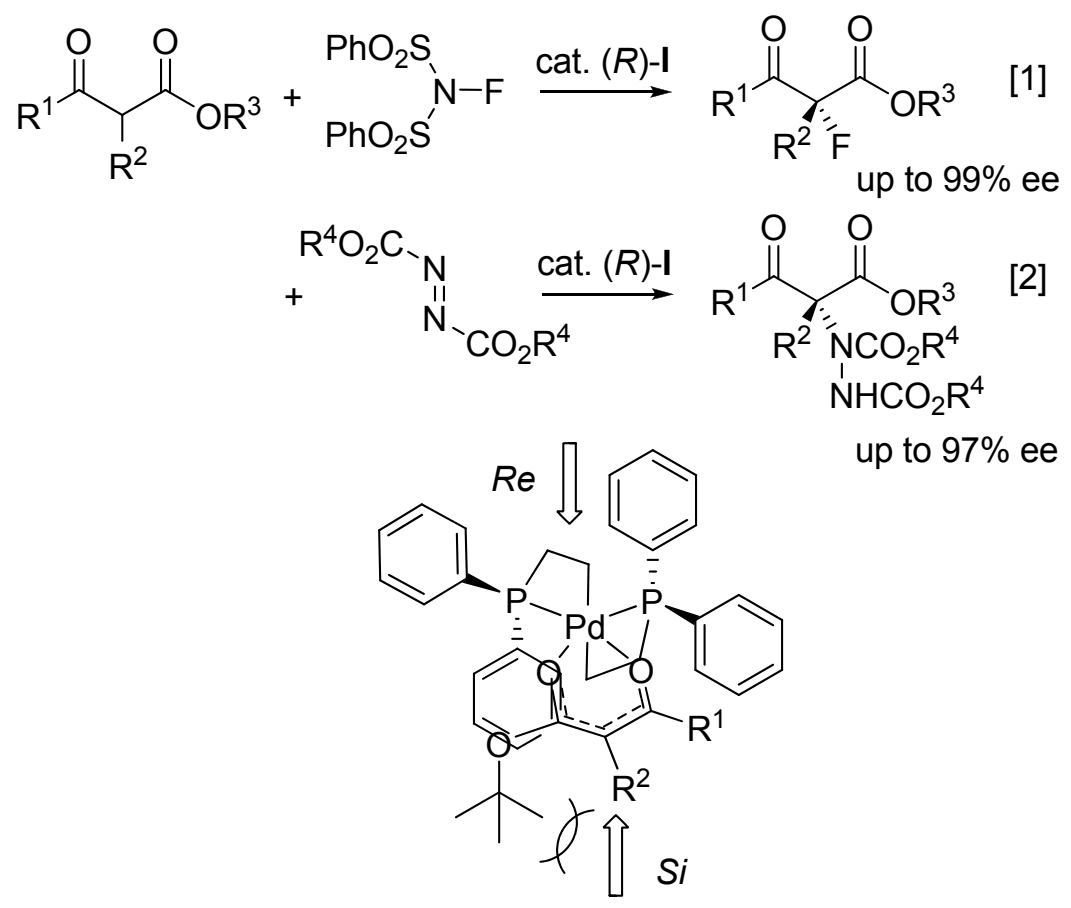

Scheme 7. Previously proposed stereochemical model for Pd-catalyzed $\alpha-$ heterofunctionalization of 1,3-ketoesters.

In light of this, it came as a great surprise to find that this does not apply to the present system. Using $(R)$-I as the catalyst, hydroxylation products containing the $S$-configuration were obtained as the preferred enantiomer, clearly defying the stereoselectivity predicted by this model. To be certain, the stereochemical assignment of $\alpha$-hydroxyl ketoesters was verified by comparing the optical rotations of our compounds to those reported in the literature, which have been based, invariably, on correlations to assignments of $(R)-(+)$ and $(S)-(-)$-configurations to 15a and 29 (figure 9) made by Davis on the basis of circular dichroism spectra. ${ }^{4}$ These assignments were verified independently in this work by calculating the electronic circular dichroism (ECD) response for 15a and 29. Using time-dependent DFT theory at the B3LYP/aug-cc-pVTZ level 
with applied continuum solvation field correction for methanol solvent (WEO Table and supporting info), the signs of the peaks match those reported, confirming the original assignment of absolute configuration of both these compounds.<smiles>CC(=O)OC1(O)CCc2ccccc2C1=O</smiles>

$(S)-(-)-15 a$<smiles>COc1ccc(OC)c2c1CC[C@](O)(OC(C)=O)C2=O</smiles>

Figure 9. Assigned stereochemistry of $\mathbf{1 5 a}$ and 29 by circular dichroism (calculated and observed).

Additionally, the absolute configuration of $\mathbf{2 5 c}$ was established recently by a crystal structure of a closely-related derivative. ${ }^{24}$ Furthermore, we obtained a single crystal of an optically pure sample of 20b, which was analyzed by X-ray crystallography (figure 10). By use of the Flack parameter $\left[x^{+}=0.00(12), x^{-}=1.04(12)\right],{ }^{33}$ it was possible to determine purely from the diffraction data that the species present in the crystal has the $S$-configuration shown.

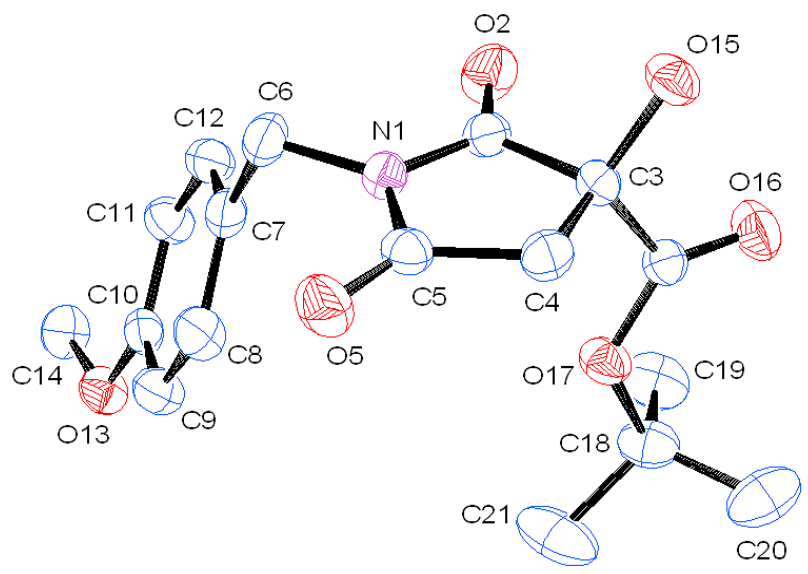

Figure 10. Molecular structure of $(S)-(+)-20 b$ determined by X-ray crystallography $(50 \%$ thermal ellipsoids).

With unequivocal validation of our stereochemical assignment, an alternate explanation is needed for the observed selectivity. A quantitative transition state model was constructed for the 
purpose (previous models had been based on non-transition state geometries obtained using molecular mechanics methods ${ }^{27}$ ). The standard DFT procedure at the B3LYP/cc-pVDZ level of theory (cc-pVDZ-pseudopotential for Pd) was enhanced with a continuum solvation model $(\mathrm{CPCM}$, solvent $=$ acetone $)$, and the geometries of the transition states for oxygen atom transfer from DMD were fully located and optimized within this model. Such an approach was necessary in order to be able to quantitatively define model systems with a dipole moment $>20 \mathrm{D}$, this magnitude resulting from ionization of the TfO-Pd to form an ion-pair complex. The energy of the resulting geometric model was then corrected for thermal and entropic effects via normal coordinate analysis to give free energies of activation $\left(\sim 11-13 \mathrm{kcal} \mathrm{mol}^{-1}\right)$, which are commensurate with the observed kinetics of these reactions. Animations of the calculated transition normal modes for the hydroxylation of substrate $10 \mathrm{c}(\mathrm{R}=t$-Bu$)$ and $10 \mathrm{~d}(\mathrm{R}=\mathrm{Me})$ by DMD are presented in the Web-enhanced Table 1, where a more complete set of geometric features can also be explored interactively. Several key features are also highlighted in figure 11.

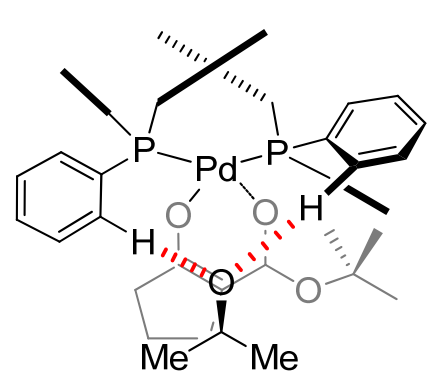

(a) $\mathrm{CH}$...O bonding to $\mathrm{DMD}$

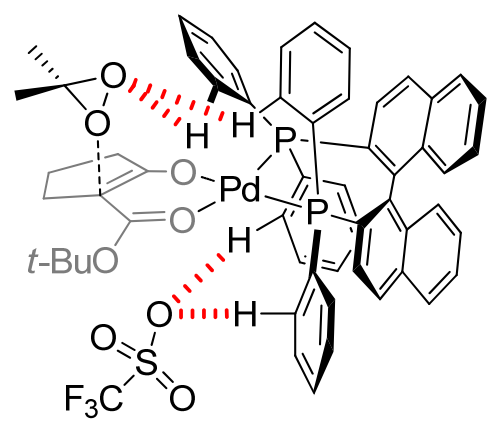

(b) $\mathrm{CH}$..O bonding to anion

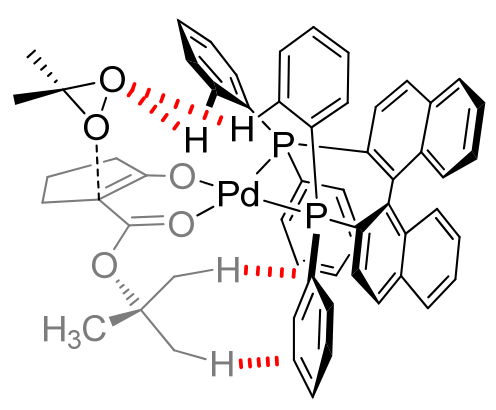

(c) $\mathrm{C}-\mathrm{H} / \pi$ interaction, $\mathrm{TS}(\mathrm{Si})$

Figure 11. Key interactions present in the favored transition state (TSSi): (a) top view depicting C-H...O interactions with DMD; (b) side view depicting C-H...O interactions with triflate anion; and (c) side view depicting C-H... $\pi$ interactions. 
The first striking difference from the previous model is the preservation of the s-trans conformation of the ester moiety; even a tert-butyl ester group can be accommodated in the plane of the chelate ring throughout the process. The oxygen transfer step occurs via a highly asynchronous spiro transition state, where the oxirane ring approaches $\mathrm{C}-2$ practically in a linear trajectory. ${ }^{34}$ The approach of oxirane on either face of the enolate is strongly directed by two C-H...O hydrogen bonds (ca. $2.6 \AA$ ), between the oxidant and the BINAP ligand (figure 11a). This causes significant distortion in the arrangement of the P-Ph groups from the 'classical' quadrant occupancy. Additionally, there are also clear interactions between the aromatic C-H bonds with the ionized triflate anion ( $c a .2 .4 \AA$ ), which resides in a pocket opposite to that of the reacting DMD reagent (figure 11b). These interactions are present in both favored and disfavored transition states. However, only in the favored transition state (TSSi), are there discernible C-H/ $\pi$ interactions between the tert-butyl substituent and the equatorial phenyl group (figure 11c). These are distinctly absent in the disfavored transition state, due to the intervention of one of the methyl substituents of the oxirane ring positioned between the ester substituent and the equatorial $\mathrm{Ph}$. Considered as the weakest form of hydrogen bonding, $\mathrm{C}-\mathrm{H} / \pi$ interactions are largely dispersive in nature, and are very probably much more common in organic and organometallic chemistry than would be apparent from many simple models. ${ }^{35}$ In the present system, this appears to be crucial for stereselectivity.

A more systematic analysis of the weak interactions in this complex system can be obtained from the atoms-in-molecule (AIM) electronic density topology of the transition state, from which a large number of bond critical points (BCPs) corresponding to weak interactions can be identified. Although one must be wary about making a connection between such a topological property of the electron density and the clear existence of an attractive interaction between two 
atomic centers, ${ }^{36}$ the presence of so many critical points in even such a relatively simple catalytic reaction centre suggests that the overall specificity of the reaction is as likely to be mediated by the overall sum of many such weak interactions, as by a few particularly strong heuristic (i.e. memorably named) ones. No accepted methodology exists for summing such a large number of diverse "weak" interactions - each of which might need individual calibration for the energy of interaction, and we do not attempt such here. Nonetheless, we recognize that many, if not most, of these interactions are indeed likely to be dispersive in nature, originating from long range correlation effects. The B3LYP-DFT procedure approximates only the short range and not the long range correlation effects. To approximately estimate the correction for the latter, we also performed single point energy evaluations using a dispersion-corrected DFT method (B97D), ${ }^{37}$ in which a simple empirical correction is added to mimic the long range dispersion effects. For the hydroxylation of the tert-butyl ester 10c, the B3LYP + solvation DFT method predicts a small bias for TSSi over the TSRe transition state for the methyl ester, but this is inverted for the t-butyl ester, which is contrary to our experimental observations. However, the B97D dispersioncorrected energies enhance the TSSi preference by $0.9 \mathrm{kcal} \mathrm{mol}^{-1}$ (methyl ester) and by $1.2 \mathrm{kcal}$ $\mathrm{mol}^{-1}$ (t-butyl ester), and with the latter correction, TSSi is now favored over TSRe for both esters. We also noted that fully re-optimizing the transition state geometries at the B97D level (rather than the single point energy evaluation procedure as noted above) led to unreasonable forming C-O bond lengths ( $>2.6 \AA$ compared to $\sim 2.2 \AA$ at the B3LYP level) and activation free energies $\left(\sim 1.7 \mathrm{kcal} \mathrm{mol}^{-1}\right.$ vs 11-13 at the B3LYP level).

Although our model represents a considerable advance in sophistication compared to previous ones, there are still aspects that will require future investigation. Thus the triflate anion (which the AIM analysis shows is not entirely passive) was optimized from an initial placement in an 
obvious pocket beneath the direction of approach of the DMD oxidant. ${ }^{38}$ A more systematic exploration of other positions has not yet been possible because of the computational cost. For the same reason, the effect of adding a further unit of triflic acid via hydrogen bonding interactions to various positions around the system has yet to be explored. Either of these refinements might potentially affect the predicted magnitude of the stereoselectivity. We did, however, observe that removing the triflate anion from the model entirely now favors the TSSi

over TSRe by $3.6 \mathrm{kcal} \mathrm{mol}^{-1}$ in free energy (B3LYP model, t-butyl ester), which hints that even the nature of the counter-ion may influence the outcome. We do nevertheless feel we have demonstrated that a fully optimized model for such a complex reaction site is now entirely possible for systems where an ion-pair is involved. Indeed, it is worth re-iterating that a fully quantum-mechanical model is essential not merely to reproduce the bond-breaking at the transition state, but the plethora of weaker interactions between the various components. ${ }^{39}$

\section{Conclusions}

Highly enantioselective Pd-catalyzed $\alpha$-hydroxylation of 1,3-ketoesters can be achieved using dicationic palladium catalyst $\mathbf{I}$ and DMD as oxidant. By extensive screening of reaction conditions, reactants and catalysts, key parameters important for stereocontrol have been revealed. With a certain combination of catalyst and oxidant, the stereoselectivity of the process can be controlled by varying the size of the ester substituent. Unusual reactivity patterns were uncovered, which was attributed to highly synergistic relationship between the catalytic intermediates and the oxidant, which has a dramatic effect on catalytic turnover.

During this work, we have also uncovered an unprecedented stereochemical pathway for the $\alpha$-hydroxylation of ketoesters, which opposes that previously observed in other $\alpha$ - 
functionalization reactions. Quantitative DFT models show that the stereoselectivity of the process is directed by a combination of $\mathrm{C}-\mathrm{H} \ldots \mathrm{O}$ and $\mathrm{C}-\mathrm{H} / \pi$ interactions, with the latter conferring facial discrimination to the hydroxylation process. As far as we are aware, this is the first time such weak intermolecular forces have been implicated in an asymmetric process.

\section{Experimental Section}

Catalytic procedures, Method A (catalyst I and DMD): A Radley's reaction tube was charged with $\beta$-ketoester $(0.123 \mathrm{mmol})$, catalyst I $(6.58 \mathrm{mg}, 6.15 \mu \mathrm{mol})$ and $\mathrm{CH}_{2} \mathrm{Cl}_{2}(0.5 \mathrm{~mL})$ and cooled to $-20{ }^{\circ} \mathrm{C}$. A solution of DMD in acetone $(0.09 \mathrm{M}, 1.64 \mathrm{~mL}, 0.147 \mathrm{mmol})$ at $-20^{\circ} \mathrm{C}$ was then added dropwise. After 30 min the mixture was concentrated, and filtered through a plug

of silica. The crude product was either judged to be pure enough by ${ }^{1} \mathrm{H}$ NMR, or purified by column chromatography.

Method B (catalyst III and 1b): A Radley's reaction tube was charged with $\beta$-ketoester $(0.123$ mmol), catalyst III $(5.51 \mathrm{mg}, 3.08 \mu \mathrm{mol})$ and $\mathrm{CH}_{2} \mathrm{Cl}_{2}(0.5 \mathrm{~mL})$. After $5 \mathrm{~min}, \mathbf{1 b}(40.7 \mathrm{mg}, 0.148$ mmol) was added and the solution stirred until judged to be complete by the starting material was consumed (TLC). The reaction mixture was then concentrated in vacuo and purified by column chromatography.

Method C (using catalyst II): A Radley's reaction tube was charged with a solution of catalyst II $(25.0 \mathrm{mg}, 24.6 \mu \mathrm{mol})$ in $\mathrm{CH}_{2} \mathrm{Cl}_{2}(0.5 \mathrm{~mL})$ and powdered molecular sieves $(4 \AA, 125$ $\mathrm{mg})$. After stirring for $30 \mathrm{~min}$, a solution of $\beta$-ketoester $(0.246 \mathrm{mmol})$ in $\mathrm{CH}_{2} \mathrm{Cl}_{2}(0.5 \mathrm{~mL})$ was added, followed by $\mathbf{1 b}(81.2 \mathrm{mg}, 0.295 \mathrm{mg})$ and the progress of the reaction was monitored (TLC). When this was completed, the reaction mixture was then filtered through a plug of celite, concentrated in vacuo and purified by column chromatography. 
Method D (ligand screening): Under a nitrogen atmosphere, a Radley's reaction tube was purged with nitrogen and charged with $\mathrm{Pd}(\mathrm{OTf})_{2} .2 \mathrm{H}_{2} \mathrm{O}^{40}(5.4 \mathrm{mg}, 12.3 \mu \mathrm{mol})$, the appropriate ligand $(13.5 \mu \mathrm{mol})$ and $\mathrm{CH}_{2} \mathrm{Cl}_{2}(0.4 \mathrm{~mL})$. After stirring for $30 \mathrm{~min}$, a solution of $7 \mathbf{a}(23.4 \mathrm{mg}$, $0.123 \mathrm{mmol})$ in $\mathrm{CH}_{2} \mathrm{Cl}_{2}(0.1 \mathrm{~mL})$ was added, followed after a further 5 min by a solution of $\mathbf{1 b}$ (40.7 $\mathrm{mg}, 0.148 \mathrm{mmol})$ in $\mathrm{CH}_{2} \mathrm{Cl}_{2}(0.1 \mathrm{~mL})$ and the solution stirred for $18 \mathrm{~h}$. The reaction mixture was then concentrated in vacuo, filtered through a plug of silica and analyzed by NMR and chiral HPLC.

Crystal data for (S)-20b: $\mathrm{C}_{17} \mathrm{H}_{21} \mathrm{NO}_{6}, M=335.35$, monoclinic, $P 2_{1}$ (no. 4), $a=6.34741(7), b$ $=10.76431(9), c=12.86992(13) \AA, \beta=102.2709(10)^{\circ}, V=859.254(15) \AA^{3}, Z=2, D_{\mathrm{c}}=$ $1.296 \mathrm{~g} \mathrm{~cm}^{-3}, \mu(\mathrm{Cu}-\mathrm{K} \alpha)=0.824 \mathrm{~mm}^{-1}, T=173 \mathrm{~K}$, colourless tablets, Oxford Diffraction Xcalibur PX Ultra diffractometer; 3386 independent measured reflections $\left(R_{\text {int }}=0.0297\right), F^{2}$ refinement, $R_{1}(\mathrm{obs})=0.0259, w R_{2}(\mathrm{all})=0.0688,3221$ independent observed absorptioncorrected reflections $\left[\left|F_{\mathrm{o}}\right|>4 \sigma\left(\left|F_{\mathrm{o}}\right|\right), 2 \theta_{\max }=145^{\circ}\right], 223$ parameters. The absolute structure of $(S)$-20b was determined by a combination of $R$-factor tests $\left[R_{1}^{+}=0.0259, R_{1}^{-}=0.0262\right]$ and by use of the Flack parameter $\left[x^{+}=0.00(12), x^{-}=1.04(12)\right]$. CCDC765842.

Acknowledgment. We thank EPSRC and Pfizer for studentship support (AMRS), and Johnson Matthey plc for the loan of Pd salts. Gifts of chiral ligands were received from Solvias, Johnson Matthey, Dow Pharma, Chirotech, Prof. Antonio Togni (ETH, Zurich), and Prof. A. Pfaltz (University of Basel). We are grateful to Prof. K. Maruoka (Kyoto University) for providing useful discussion and reference 24 . 
Supporting Information Available. Web-enhanced table, CIF file, experimental procedure, characterization data, X-ray crystallographic data and copies of ${ }^{1} \mathrm{H}$ and ${ }^{13} \mathrm{C}$ NMR spectra.

\section{References}

1. Rubottom, G. M.; Vazquez, M. A.; Pelegrin, D. R. Tetrahedron Lett. 1974, 15, 4319.

2. Christoffers, J.; Baro, A.; Werner, T. Adv. Synth. Catal. 2004, 346, 143.

3. Davis, F. A.; Chen, B. C. Chem. Rev. 1992, 92, 919.

4. $\quad$ Davis, F. A.; Liu, H.; Chen, B.-C.; Zhou, P. Tetrahedron 1998, 54, 10481.

5. See for example: (a) Hauser, F. M.; Xu, Y. J. Org. Lett. 1999, 1, 335; (b) Davies, S. G.; Epstein, S. W.; Garner, A. C.; Ichihara, O.; Smith, A. D. Tetrahedron-Asymmetry 2002, 13, 1555; (c) Abraham, E.; Candela-Lena, J. I.; Davies, S. G.; Georgiou, M.; Nicholson, R. L.; Roberts, P. M.; Russell, A. J.; Sanchez-Fernandez, E. M.; Smith, A. D.; Thomson, J. E., Tetrahedron-Asymmetry 2007, 18, 2510; (d) Tagami, K.; Nakazawa, N.; Sano, S.; Nagao, Y. Heterocycles 2000, 53, 771.

6. Toullec, P. Y.; Bonaccorsi, C.; Mezzetti, A.; Togni, A. PNAS 2004, 101, 5810.

7. Ishimaru, T.; Shibata, N.; Nagai, J.; Nakamura, S.; Toru, T.; Kanemasa, S. J. Am. Chem. Soc. 2006, 128, 16488.

8. Taylor, E. G.; Birch, L. US Patent WO03/040083, PCT/US02/35615 (2003).

9. Acocella, M. R.; Mancheno, O. G.; Bella, M.; Jorgensen, K. A. J. Org. Chem. 2004, 69, 8165. 
10. Lu, M.; Zhu, D.; Lu, Y. P.; Zeng, X. F.; Tan, B.; Xu, Z. J.; Zhong, G. F. J. Am. Chem. Soc. 2009, 131, 4562.

11. Smith, A. M. R.; Billen, D.; Hii, K. K. Chem. Commun. 2009, 3925.

12. Hii, K. K. Pure Appl. Chem. 2006, 78, 341.

13. Guino, M.; Phua, P. H.; Caille, J. C.; Hii, K. K. J. Org. Chem. 2007, 72, 6290.

14. Phua, P. H.; Mathew, S. P.; White, A. J. P.; de Vries, J. G.; Blackmond, D. G.; Hii, K. K. Chem-Eur. J. 2007, 13, 4602.

15. Sodeoka, M.; Hamashima, Y. Bull. Chem. Soc. Jpn. 2005, 78, 941.

16. Hamashima, Y.; Suzuki, T.; Takano, H.; Shimura, Y.; Tsuchiya, Y.; Moriya, K.; Goto, T.; Sodeoka, M. Tetrahedron 2006, 62, 7168.

17. Hamashima, Y.; Sodeoka, M. Synlett 2006, 1467.

18. Kang, Y. K.; Kim, D. Y. Tetrahedron Lett. 2006, 47, 4565.

19. Hydrogen bonding can counteract steric effects, leading to opposite selectivities in epoxidation reactions, see: (a) Adam, W.; Pastor, A.; Peters, K.; Peters, E. M. Org. Lett. 2000, 2, 1019; (b) Adam, W.; Zhang, A. M. Eur. J. Org. Chem. 2004, 147.

20. Phua, P. H.; White, A. J. P.; de Vries, J. G.; Hii, K. K. Adv. Synth. Catal. 2006, 348, 587.

21. Control experiments performed using $\mathrm{Pd}(\mathrm{OTf})_{2} \cdot 2 \mathrm{H}_{2} \mathrm{O}$ in the absence of ligands led to a capricious reaction, with little product formation. 
22. For example, see: (a) Li, D.; Schröder, K.; Bitterlich, B.; Tse, M. K.; Beller, M. Tetrahedron Lett. 2008, 49, 5976; (b) Watanabe, T.; Ishikawa, T. Tetrahedron Lett. 1999, 40, 7795; (c) Christoffers, J. J. Org. Chem. 1999, 64, 7668; (d) Christoffers, J.; Werner, T.; Unger, S.; Frey, W. Eur. J. Org. Chem. 2003, 425.

23. Hashimoto, T.; Sasaki, K.; Fukumoto, K.; Murase, Y.; Ooi, T.; Maruoka, K. Synlett 2009, 661.

24. Hashimoto, T.; Sasaki, K.; Fukumoto, K.; Murase, Y.; Abe, N.; Ooi, T.; Maruoka, K. Chem.-Asian J. 2010, in press, DOI: 10.1002/asia.200900344.

25. Fujii, A.; Hagiwara, E.; Sodeoka, M. J. Am. Chem. Soc. 1999, 121, 5450.

26. Attempts to isolated complex IV were unsuccessful.

27. Hamashima, Y.; Hotta, D.; Umebayashi, N.; Tsuchiya, Y.; Suzuki, T.; Sodeoka, M. Adv. Synth. Catal. 2005, 347, 1576.

28. Nama, D.; Pregosin, P. S.; Albinati, A.; Rizzato, S. Organometallics 2007, 26, 2111.

29. Sodeoka, M.; Hamashima, Y. Pure Appl. Chem. 2006, 72, 477.

30. Sodeoka, M.; Hamashima, Y. Chem. Commun. 2009, 5787.

31. Attempts to isolate the complex were unsuccessful.

32. Suzuki, T.; Goto, T.; Hamashima, Y.; Sodeoka, M. J. Org. Chem. 2007, 72, 246.

33. Flack, H. D. Acta Cryst. A 1983, 39, 876.

34. Dufert, A.; Werz, D. B. J. Org. Chem. 2008, 73, 5514. 
35. Tsuzuki, S.; Fujii, A. Phys. Chem. Chem. Phys. 2008, 10, 2584.

36. Bader, R. F. W. J. Phys. Chem. A 2009, 113, 10391.

37. Grimme, S. J. Comp. Chem. 2006, 27, 1787.

38. Quinonero, D.; Deya, P. M.; Carranza, M. P.; Rodriguez, A. M.; Jalon, F. A.; Manzano, B. R. Dalton Trans. 2010, 39, 794.

39. The DFT-model constructed involved up to 126 atoms, specified by up to 1323 basis functions, and with both first and second energy derivatives analytically computed within a continuum solvent field. These are close to the current (2009) practical limits, although there is every reason to expect that extending the models to around 150 atoms at this level will be feasible in the near future, as will the ability for more extensive exploration of the ion-pair and solvent spaces.

40. Murata, S.; Ido, Y. Bull. Chem. Soc. Jpn. 1994, 67, 1746. 